\title{
Quantifying stochastic resonance: theory versus experiment
}

\author{
Mykhaylo Evstigneev $^{1}$, Peter Reimann ${ }^{1}$, Carmen Schmitt $^{2}$ and \\ Clemens Bechinger ${ }^{2}$ \\ 1 Theoretische Physik, Universität Bielefeld, Universitätsstraße 25, 33615 Bielefeld, Germany \\ 2 Physikalisches Institut, Universität Stuttgart, Pfaffenwaldring 57, 70569 Stuttgart, Germany
}

\begin{abstract}
We discuss different quantifiers of stochastic resonance (SR) and how far they are mathematically related with each other. Specifically, we address bona fide SR in terms of the areas of the hysteresis loops and of the first peaks in the residence time distributions. We demonstrate a surprisingly good agreement of these two SR quantifiers experimentally for colloidal particles in periodically modulated laser traps. A simple theoretical model is established, which reproduces the experimental observations very well.
\end{abstract}

(Some figures in this article are in colour only in the electronic version)

\section{Introduction}

Stochastic resonance (SR) refers to the enhanced sensitivity of a nonlinear system to a weak periodic forcing in the presence of an optimal amount of noise [1]. Such a mechanism may play a role in various natural systems like neurophysiology, ice ages, or geomagnetism, and may also be of interest in the context of optimizing signal detectors of various types. Since the phenomenon is very broad, there is still no general agreement about the precise conditions for its occurrence, its physical meaning, and even its defining characteristic signature; see e.g. [2-8]. A considerable variety of different quantifiers for SR has been introduced in the course of time, motivated by the same general ideas, but leading to different quantitative conclusions regarding the occurrence of SR in a given physical system.

At the focus of our present work are SR effects in colloidal suspensions under the action of periodically modulated laser traps. In section 2 we introduce the general framework and derive some relations between different SR quantifiers in the linear response regime, whose implications are then discussed in section 3. In section 4 we put forward a very simple theoretical model for the approximate description of the experiments in section 5. The predictions of this model are then compared with experimental observations, yielding very good quantitative agreement. These measurements together with the description of the experimental set-up and the justification of the theoretical model are the content of section 5. Since the 
noise intensity cannot be changed in the experiment, we focus on optimal signal enhancement phenomena upon variation of the driving frequency — so called 'bona fide SR'. Two prominent quantifiers of SR in such a case are based on residence time distributions [2-4, 9, 10] and hysteresis loop areas [11-19], as discussed in detail in sections 2-5. Our summary and conclusions are contained in section 6.

\section{Linear response theory}

In the following we systematically review relations between three prominent quantifiers of SR, namely the signal-to-noise ratio (SNR), the spectral power amplification (SPA), and the hysteresis loops area (HLA), in the linear response regime of weak periodic driving. Most of these relations are well known, but some of them we have not see before in the literature. In this section we focus on the general framework and the mathematics, while the physical discussion is postponed to the subsequent section 3 .

\subsection{Framework}

We consider an arbitrary system whose equilibrium probability density is of Boltzmann form

$$
W_{\text {eq }}(\phi)=Z^{-1} \exp \left\{-H(\phi) / k_{\mathrm{B}} T\right\},
$$

for a suitably defined Hamiltonian $H(\phi)$ on a phase space with elements $\phi$.

Next we assume that the system is subject to a weak, time-dependent perturbation, amounting to a Hamiltonian of the form $H(\phi)-x(\phi) f(t)$. In the context of SR, $x(\phi)$ is typically one of the system's spatial coordinates and $f(t)$ an external force. In fact, by means of a canonical transformation one can usually reduce the problem to this special case.

The time evolution of the perturbed system is required to be Markovian, i.e. its future only depends on the present, not on the past. Furthermore, we take for granted that the long time limit of the probability density $W(\phi, t)$ is unique, i.e. independent of the initial condition, for any given perturbation $f(t)$ of the system. It follows that this long time limit is the Boltzmann distribution (1) in the absence of driving, and that for a periodic driving $f(t)$ the probability density will be asymptotically periodic as well. Moreover, it follows that the dynamics is mixing in the sense that the ensemble averaged autocorrelation of the system coordinate satisfies

$$
\langle x(t) x(t+s)\rangle \rightarrow\langle x(t)\rangle\langle x(t+s)\rangle \quad \text { for } s \rightarrow \infty,
$$

where $x(t):=x(\phi(t))$.

We remark that the above assumptions about the dynamics are rather weak and thus our subsequent considerations apply to a very large class of different systems.

All in all, we may say that two different 'input forces' are acting simultaneously on the system coordinate $x$, namely thermal fluctuations $\xi(t)$ and the external driving force $f(t)$. In this sense, the 'input signal' of the system is given by

$$
x_{\text {in }}(t):=\xi(t)+f(t),
$$

where $\xi(t)$ must be a white noise (uncorrelated in time) due to our above assumption of a Markovian dynamics.

The central question in SR is how the system coordinate $x(t)$, considered as the 'output' of the nonlinear, noisy dynamics, responds to this input, in particular the 'sensitivity' of this response in the case of a weak, periodic forcing $f(t)$. 


\subsection{Fluctuation-dissipation theorem}

The response of the ensemble averaged coordinate to the weak external perturbation $f(t)$ can be written by means of a functional expansion in the form

$$
\langle x(t)\rangle=\langle x\rangle_{\mathrm{eq}}+\int_{0}^{\infty} \mathrm{d} t^{\prime} R\left(t^{\prime}\right) f\left(t-t^{\prime}\right)+\mathcal{O}\left(f^{2}(t)\right)
$$

where $\langle\cdots\rangle_{\text {eq }}$ means averaging with respect to the equilibrium probability density (1). The linear response function $R\left(t^{\prime}\right)$ describes the effect of the driving field $f(t)$ at the previous moment of time $t-t^{\prime}$ on the system's average coordinate at the present time $t$. Because of causality, the driving field at some future time moment $t+t^{\prime}>t$ cannot have any effect on the system's properties at time $t$, thus $R\left(t^{\prime}\right)=0$ for $t^{\prime}<0$.

A central point of the functional expansion (4) is the fact that the linear response function $R(t)$ in general depends on everything else, but not on the perturbation $f(t)$. In particular, $f(t)$ need not be periodic in time. In order to evaluate $R(t)$, we thus can focus on the evolution of the system's average coordinate under the action of a suitably chosen, specific perturbation $f(t)$, namely a step function

$$
f_{\text {step }}(t):=\epsilon \Theta(-t)
$$

of small amplitude $\epsilon$, where $\Theta(t):=\int_{-\infty}^{t} \mathrm{~d} t^{\prime} \delta\left(t^{\prime}\right)$ is the Heaviside step function. In other words, an external bias $\epsilon$ is switched on in the infinitely remote past $t=-\infty$ and switched off at time $t=0$. Thus the system is allowed to equilibrate with the bias for all finite times $t<0$, i.e. the probability density at times $t \leqslant 0$ is Boltzmannian:

$$
W_{\epsilon}(\phi)=\underset{\exp \left[-(H(\phi)-\epsilon x(\phi)) / k_{\mathrm{B}} T\right]}{\int \mathrm{d} \phi^{\prime} \exp \left[-\left(H\left(\phi^{\prime}\right)-\epsilon x\left(\phi^{\prime}\right)\right) / k_{\mathrm{B}} T\right]}=W_{\mathrm{eq}}(\phi)\left(\begin{array}{c}
1+\epsilon(\phi)-\langle x\rangle_{\mathrm{eq}} \\
k_{\mathrm{B}} T
\end{array}\right)+\mathcal{O}\left(\epsilon^{2}\right),
$$

where the argument $t$ in $\langle x\rangle_{\mathrm{eq}}$ is omitted since this average is time independent.

Next we introduce the conditional probability $P\left(\phi, t \mid \phi_{0}, t_{0}\right)$ to find the system in the state $\phi$ at the moment $t \geqslant 0$, provided that the system's state at time $t_{0}<t$ was $\phi_{0}$. Due to our assumption that the dynamics is Markovian, $P\left(\phi, t \mid \phi_{0}, t_{0}\right)$ fully determines the time evolution according to

$$
W(\phi, t)=\int \mathrm{d} \phi_{0} P\left(\phi, t \mid \phi_{0}, t_{0}\right) W\left(\phi_{0}, t_{0}\right)
$$

Since the bias $\epsilon$ is switched off at time $t=0$, we can conclude that

$$
\int \mathrm{d} \phi_{0} P\left(\phi, t \mid \phi_{0}, 0\right) W_{\mathrm{eq}}\left(\phi_{0}\right)=W_{\mathrm{eq}}(\phi)
$$

for all $t \geqslant 0$. Hence the average $\langle x(t)\rangle$ evolves according to

$$
\begin{aligned}
\langle x(t)\rangle_{\text {step }}= & \int \mathrm{d} \phi x(\phi) W(\phi, t)=\int \mathrm{d} \phi x(\phi) \int \mathrm{d} \phi_{0} P\left(\phi, t \mid \phi_{0}, 0\right) W\left(\phi_{0}, 0\right) \\
& =\int \mathrm{d} \phi x(\phi) \int \mathrm{d} \phi_{0} P\left(\phi, t \mid \phi_{0}, 0\right) W_{\mathrm{eq}}\left(\phi_{0}\right)\left(1+\epsilon \begin{array}{c}
x\left(\phi_{0}\right)-\langle x(\phi)\rangle_{\mathrm{eq}} \\
k_{\mathrm{B}} T
\end{array}\right)+\mathcal{O}\left(\epsilon^{2}\right) \\
& =\langle x\rangle_{\mathrm{eq}}+\epsilon \begin{array}{c}
\langle x(0) x(t)\rangle_{\mathrm{eq}}-\langle x\rangle_{\mathrm{eq}}^{2} \\
k_{\mathrm{B}} T
\end{array} \mathcal{O}^{2}\left(\epsilon^{2}\right),
\end{aligned}
$$

where $\langle x(0) x(t)\rangle_{\mathrm{eq}}$ is the coordinate autocorrelation function in the absence of driving. On the other hand, for our step function (5) the average $\langle x(t)\rangle_{\text {step }}$ at time $t$ is given according to (4) by

$$
\langle x(t)\rangle_{\mathrm{step}}=\langle x\rangle_{\mathrm{eq}}+\epsilon \int_{t}^{\infty} \mathrm{d} t^{\prime} R\left(t^{\prime}\right)+\mathcal{O}\left(\epsilon^{2}\right)
$$


so that the linear response function is related to the coordinate autocorrelation function as

$$
R(t)=-\begin{array}{cc}
1 \mathrm{~d}\langle x(t)\rangle_{\mathrm{step}} \\
\epsilon & \mathrm{d} t
\end{array}=-\begin{array}{cc}
1 & \mathrm{~d}\langle x(0) x(t)\rangle_{\mathrm{eq}} \\
k_{\mathrm{B}} T & \mathrm{~d} t
\end{array}
$$

This relation is known as the fluctuation-dissipation theorem [20, 21].

\subsection{Susceptibility}

Introducing the susceptibility

$$
\chi(\omega):=\int_{0}^{\infty} \mathrm{d} t R(t) \mathrm{e}^{-\mathrm{i} \omega t}
$$

into the fluctuation-dissipation theorem (11), integrating by parts, and considering (2) yields for the imaginary part of the susceptibility the relation

$$
\operatorname{Im} \chi(\omega)=-{ }_{k_{\mathrm{B}} T}^{\omega} \int_{0}^{\infty} \mathrm{d} t\langle x(0) x(t)\rangle_{\mathrm{eq}} \cos (\omega t) .
$$

The real part of the susceptibility can be found from the imaginary part, and vice versa, using the Kramers-Kronig relations [22]:

$$
\begin{aligned}
& \operatorname{Re} \chi(\omega)=-{ }_{\pi}^{1} P \int_{-\infty}^{\infty} \mathrm{d} \omega^{\prime} \begin{array}{c}
\operatorname{Im} \chi\left(\omega^{\prime}\right) \\
\omega^{\prime}-\omega
\end{array}, \\
& \operatorname{Im} \chi(\omega)={ }_{\pi}^{1} P \int_{-\infty}^{\infty} \mathrm{d} \omega^{\prime} \operatorname{Re} \chi\left(\omega^{\prime}\right) \\
& \omega^{\prime}-\omega
\end{aligned}
$$

where the symbol ' $P$ ' indicates that the integrals are evaluated in the sense of the principal value. Moreover, for $\omega=0$ we can infer from (2), (11), and (12) that

$$
\chi(0)=\frac{\left\langle x^{2}\right\rangle_{\mathrm{eq}}-\langle x\rangle_{\mathrm{eq}}^{2}}{k_{\mathrm{B}} T}
$$

\subsection{SR quantifiers}

For an arbitrary periodic driving $f(t)$ of frequency $\Omega$ with Fourier decomposition

$$
f(t)=\sum_{n=1}^{\infty} A_{n} \cos \left(n \Omega t+\varphi_{n}\right)
$$

the driven average coordinate from (4) evolves according to

$$
\langle x(t)\rangle=\langle x\rangle_{\mathrm{eq}}+\operatorname{Re} \sum_{n=1}^{\infty} \chi(n \Omega) A_{n} \mathrm{e}^{\mathrm{i}\left(n \Omega t+\varphi_{n}\right)}+\mathcal{O}\left(f^{2}(t)\right) .
$$

Within linear response (weak driving), this yields

$$
\langle x(t)\rangle=\langle x\rangle_{\mathrm{eq}}+\sum_{n=1}^{\infty}|\chi(n \Omega)| A_{n} \cos \left(n \Omega t+\varphi_{n}+\delta_{n}\right),
$$

with

$$
\begin{aligned}
& |\chi(n \Omega)|=\sqrt{ }[\operatorname{Re} \chi(n \Omega)]^{2}+[\operatorname{Im} \chi(n \Omega)]^{2}, \\
& \tan \delta_{n}=\operatorname{Im} \chi(n \Omega) / \operatorname{Re} \chi(n \Omega) .
\end{aligned}
$$

The spectral density of coordinate fluctuations in the absence of driving is defined as

$$
S_{\mathrm{eq}}(\omega):=\int_{-\infty}^{\infty} \mathrm{d} t\langle x(0) x(t)\rangle_{\mathrm{eq}} \mathrm{e}^{-\mathrm{i} \omega t} .
$$


Taking into account that the equilibrium correlation function is an even function of time, $\langle x(0) x(-t)\rangle_{\mathrm{eq}}=\langle x(0) x(t)\rangle_{\mathrm{eq}}$, we can infer from (13) and (22) the following relation between spectral density and susceptibility:

$$
S_{\mathrm{eq}}(\omega)=-2 k_{\mathrm{B}} T \begin{gathered}
\operatorname{Im} \chi(\omega) \\
\omega
\end{gathered} .
$$

By definition, the spectral power amplification (SPA) is given by the ratio of the power of the driven oscillations in (19) to that of the driving signal in (17) at the driving frequency $\Omega$, i.e.

$$
\mathrm{SPA}:=|\chi(\Omega)|^{2} \text {. }
$$

Moreover, on the $f(t)-\langle x(t)\rangle$ plane, the hysteresis loop according to (19) and (17) roughly takes the form of an ellipse whose hysteresis loop area

$$
\text { HLA }:=\int_{t=0}^{2 \pi / \Omega} \mathrm{d} f(t)\langle x(t)\rangle
$$

is given by

$$
\mathrm{HLA}=\pi \sum_{n=1}^{\infty} n A_{n}^{2}|\chi(n \Omega)| \sin \delta_{n}=\pi \sum_{n=1}^{\infty} n A_{n}^{2} \operatorname{Im} \chi(n \Omega) .
$$

Note that the area of the hysteresis loop has the physical meaning of work produced by the system per period; this work is negative since $\delta_{n} \in(-\pi, 0)$ in (19) and (21).

Our final task is to express the signal-to-noise ratio $(S N R)$ in terms of the susceptibility. Let us first focus on the correlation function $\langle x(t) x(t+s)\rangle$ in the presence of driving. In contrast to the case of the undriven system, it now depends explicitly on the time $t$. Therefore, we introduce the quasistationary correlation function by averaging with respect to this argument:

$$
C(s):=\frac{\Omega}{2 \pi} \int_{0}^{2 \pi / \Omega} \mathrm{d} t\langle x(t) x(t+s)\rangle .
$$

From (2) and (19) it follows that $C(s)-\langle x\rangle_{\text {eq }}^{2}$ converges for large $s$ towards the periodic function

$$
C_{\mathrm{coh}}(s)=\frac{1}{2} \sum_{n=1}^{\infty}|\chi(n \Omega)|^{2} A_{n}^{2} \cos (n \Omega s)
$$

up to corrections of the order $A^{4}$. Hence, one can rewrite [23] the correlation function as a sum of a coherent and an incoherent contribution

$$
C(s)=C_{\mathrm{inc}}(s)+C_{\mathrm{coh}}(s) .
$$

By definition, the incoherent part approaches $\langle x\rangle_{\mathrm{eq}}^{2}$ for $s \rightarrow \infty$. Its leading order behaviour, i.e. neglecting corrections of the order $A^{2}$, follows by letting $A \rightarrow 0$, yielding

$$
C_{\text {inc }}(s)=\langle x(0) x(s)\rangle_{\mathrm{eq}} .
$$

Correspondingly, the power spectrum of the driven system

$$
S(\omega):=\int_{-\infty}^{\infty} \mathrm{d} s C(s) \mathrm{e}^{-\mathrm{i} \omega s}
$$

can be rewritten by means of (22), (28)-(31) as

$$
S(\omega)=S_{\mathrm{eq}}(\omega)+\pi \sum_{n=1}^{\infty}|\chi(n \Omega)|^{2} A_{n}^{2}[\delta(\omega-n \Omega)+\delta(\omega+n \Omega)] / 2 .
$$


By definition [1], the signal-to-noise ratio (SNR) is given by

$$
\mathrm{SNR}:=\frac{\lim _{\delta \omega \rightarrow 0} \int_{\Omega-\delta \omega}^{\Omega+\delta \omega} \mathrm{d} \omega S(\omega)}{S_{\mathrm{eq}}(\Omega)} .
$$

Taking into account (23) and (32) we finally obtain

$$
\mathrm{SNR}=-\begin{array}{r}
\pi \Omega|\chi(\Omega)|^{2} A_{1}^{2} \\
4 k_{\mathrm{B}} T \operatorname{Im} \chi(\Omega)
\end{array} .
$$

\section{Discussion of various SR quantifiers}

The upshot of the preceding section is the following relation between signal-to-noise ratio (SNR), hysteresis loop area (HLA), and spectral power amplification (SPA) for a weak, purely harmonic driving

$$
f(t)=A \cos (\Omega t)
$$

of frequency $\Omega$ and (small) amplitude $A$ (linear response regime):

$$
\mathrm{SNR} \times \mathrm{HLA}=-\mathrm{SPA} \pi^{2} A^{4} \Omega
$$

as can be inferred from (24), (26) and (34). Hence, two of these three common quantifiers of SR determine the third one. We also recall that the HLA represents work produced by the system per period and is therefore negative. This relation is valid for a very large class of different systems as specified in section 2.1.

Often, a non-monotonic, resonance-type behaviour of one of those three quantities is considered as the defining characteristic signature of SR. In many cases, one finds that either all three of them exhibit such a maximum or all three of them are monotonic functions of the temperature. However, in general it is quite plausible and indeed confirmed in typical examples that the maximizing temperatures are different in the three cases. Moreover, there may also be cases where some of the three quantities behave monotonically and some non-monotonically for the same physical system.

Beyond the linear response regime, no generalization of the relation (36) between SNR, SPA, and HLA is known, but their generic quantitative and possibly even qualitative disagreement remains the same.

If the driving frequency $\Omega$ is much smaller than any other characteristic frequency of the system, the SPA can be readily evaluated in terms of the first two moments $\langle x\rangle_{\mathrm{eq}},\left\langle x^{2}\right\rangle_{\mathrm{eq}}$ of the unperturbed system according to (1), (16) and (24). For suitably chosen system Hamiltonians $H(\phi)$ one thus recovers an SR-type maximum of SPA as a function of temperature, but also anti-SR-type minima as well as several extrema (multiple SR), and this even in monostable systems without any clear-cut 'threshold' [8].

In the context of signal detection and enhancement, an interesting quantity is the so-called 'gain', defined as the ratio between the 'output' SNR (33) of the system through its coordinate $x$, and the corresponding 'input' SNR, associated with the total external signal (3), which acts as a force on the system coordinate $x$. Since the thermal fluctuations are given by white (uncorrelated) Gaussian noise $\xi(t)$, one readily finds for the input SNR the result

$$
\mathrm{SNR}_{\mathrm{in}}=\pi A_{1}^{2} / 4 k_{\mathrm{B}} T \text {. }
$$

Within linear response, the gain, i.e. the ratio between (34) and (37), is always smaller than unity as demonstrated in detail in [24]. On the other hand, beyond linear response and suitably chosen anharmonic driving (17), gains exceeding unity have been exemplified in [25]. 
Considered as a function of the driving frequency $\Omega$, SNR and SPA exhibit a monotonic behaviour for typical systems of interest, while HLA still shows a resonance-type nonmonotonicity, referred to as bona fide $S R$. We remark that considered as functions of $\Omega$ each of the three quantifiers completely fixes the other two thanks to the Kramers-Kronig relations (14) and (15) in combination with (24), (26) and (34).

A further popular quantifier of SR, sharing the bona fide property with HLA, is residence time distributions as discussed in more detail in the next section. To our knowledge, no general relation between this quantity and the three previously discussed SR quantifiers exists in the literature, nor were we able to derive such a relation.

While each of the above mentioned four SR quantifiers is based on the same qualitative physical picture and can be motivated by strong arguments, each of them has also its own shortcomings and unwanted features. E.g. SNR and SPA both lack the bona fide property and admit SR in monostable systems [8] as well as in any collective system near a critical phase transition point.

The shortcoming of the residence time distributions is the survival of a bona fide SR peak even in the absence of any external driving [2]. On the other hand, it is clear that HLA tends to zero both for asymptotically fast and slow external driving in basically any system, while assuming a finite value for finite driving frequency. In other words, bona fide SR in terms of HLA will be observed in basically every system. A possible way out might be to consider not the work per period, i.e. HLA itself, but rather the power absorbed by the system, i.e. HLA times driving frequency $\Omega$.

\section{Theoretical model and predictions}

In this section we exemplify bona fide SR and its two quantifiers-based on hysteresis loops and residence times, respectively_by way of a particularly simple model. The predictions for these two quantifiers will be shown in the following section 5 to agree quite well with experimental observations, thus justifying a posteriori our specific choice of the model and the parameters in this section.

\subsection{Model}

Our aim is to establish a theoretical model which quantitatively reproduces the experimental observations (see section 5) reasonably well and otherwise is as simple as possible, i.e. with a minimal number of fit parameters.

In a first step, we describe the dynamics of a colloidal particle (see section 5.1) in water as Brownian motion. Owing to the experimental realization, vertical particle fluctuations can be safely ignored and the motion of the particle is confined to a plane.

Within the plane the particle is subjected to a symmetric double well potential created by optical tweezers (see section 5.1). Our next assumption is that the problem can further be reduced to an approximate effectively one-dimensional dynamics in a (possibly renormalized) symmetric double-well potential in one dimension of the specific form

$$
U(x)=\Delta U\left[\left(x / x_{0}\right)^{2}-1\right]^{2}
$$

with two fit parameters, representing the effective potential barrier $\Delta U$ between the two symmetric potential wells at $\pm x_{0}$. The experimental justification of this approximation will be provided in section 5.2 together with the estimates

$$
\begin{aligned}
& \Delta U=2.9 k_{\mathrm{B}} T, \\
& x_{0}=0.8 \mu \mathrm{m},
\end{aligned}
$$


where the ambient temperature of the experiment is

$$
T=292 \mathrm{~K} \text { (room temperature). }
$$

As detailed in section 5.2, the intensities of the two laser traps are sinusoidally modulated in counterphase. In our model, the resulting effect on the colloidal particle is approximately described by a spatially homogeneous and temporally harmonic force (35) with frequency $\Omega$ and amplitude $A$, estimated in section 5.2 as

$$
A=1.9 k_{\mathrm{B}} T / x_{0} .
$$

Given the above approximations for the light forces, we make the following ansatz for the Brownian dynamics of the particle along the $x$-axis:

$$
\eta \dot{x}(t)=-U^{\prime}(x(t))+f(t)+\xi(t),
$$

where $\eta$ accounts for dissipation effects by Stokes friction and where thermal noise effects are modelled by unbiased white Gaussian noise $\xi(t)$, correlated according to the fluctuation dissipation theorem of the second kind [26]

$$
\langle\xi(t) \xi(s)\rangle=2 \eta k_{\mathrm{B}} T \delta(t-s) .
$$

The specific ansatz for friction and noise in (43) and (44) as well as neglecting inertia effects of the form $m \ddot{x}(t)$ (overdamped limit) represents the most widely studied model in the context of SR [1] and is well established for colloidal particles under the experimental conditions from section 5.

The only remaining model parameter in (43) is the friction coefficient $\eta$. A first estimate is provided by Stokes formula $\eta=6 \pi v r$, where $r$ is the particle radius and $v$ the viscosity of the ambient liquid (pure water). Using $r=0.79 \mu \mathrm{m}$ (see section 5.2), equation (41), and $v=0.0010 \mathrm{~N} \mathrm{~s} \mathrm{~m}^{-2}$ (water at room temperature) this yields $D=\eta / k_{\mathrm{B}} T \simeq 0.27 \mu \mathrm{m}^{2} \mathrm{~s}^{-1}$ for the diffusion coefficient $D$ equivalent to $\eta$. However, this approximation is only valid in the absence of hydrodynamic interactions between the particle and the sample wall, which, however, is not justified at the small particle-wall separations in our case. Hence, we experimentally determined the average transition time between the two symmetric potential wells, i.e. in the absence of the periodic driving, and reproduced the experimental finding of $22 \mathrm{~s}$ (see section 5.3) by numerically solving the model dynamics (43) and adapting $\eta$, yielding the estimate

$$
D=k_{\mathrm{B}} T / \eta \simeq 0.24 \mu \mathrm{m}^{2} \mathrm{~s}^{-1},
$$

for the diffusion coefficient $D$ equivalent to $\eta$, which is indeed somewhat smaller than the above naive estimate.

\subsection{Residence times and hysteresis loops}

In order to determine residence times, we introduce the two symmetric thresholds

$$
x_{ \pm}= \pm 0.6 \mu \mathrm{m}
$$

one for each potential well. The times between subsequent first crossings of the two thresholds give rise to the so called residence time distribution $P\left(t_{\text {res }}\right)$.

In figure 1 numerically determined residence time distributions $P\left(t_{\text {res }}\right)$ are shown for the model dynamics (35), (38)-(46) for various values of the driving frequency $\Omega$.

The peaks of the residence time distribution $P\left(t_{\text {res }}\right)$ reflect a kind of synchronization with the external driving $f(t)$ of period $\tau=2 \pi / \Omega$, and therefore are approximately located at 

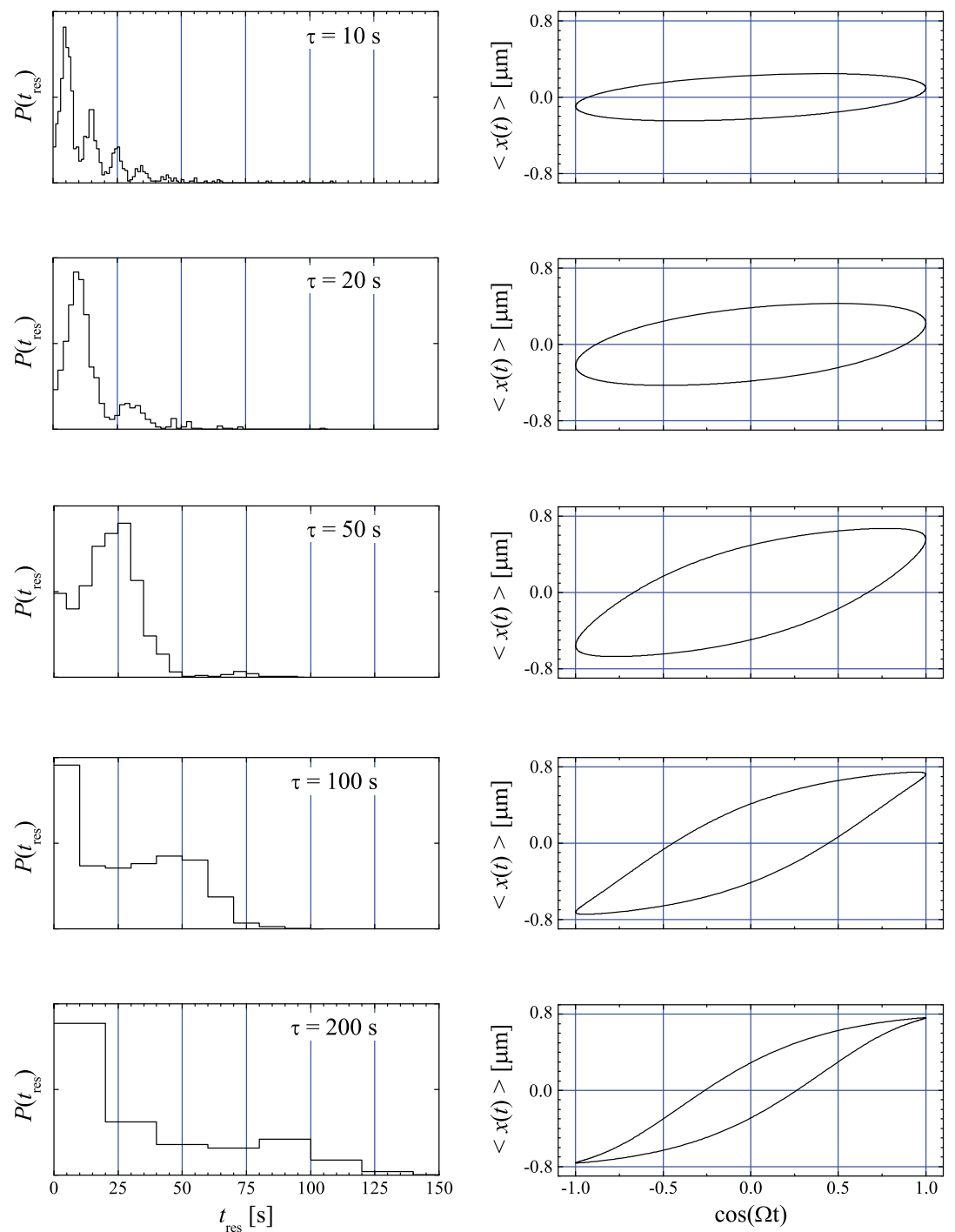

Figure 1. Residence time distributions $P\left(t_{\text {res }}\right)$ (histograms) and hysteresis loops (parametric plots of $\cos (\Omega t)=f(t) / A$ versus $\langle x(t)\rangle$ with $t$ as parameter) for five different driving periods $\tau=2 \pi / \Omega$. From top to bottom: $\tau=10,20,50,100,200 \mathrm{~s}$. The residence times have been obtained by numerical simulations of the Langevin equation (35), (38)-(46) and the averages $\langle x(t)\rangle$ by numerical solutions of the equivalent Fokker-Planck equation.

$t=(1 / 2+n) \tau, n=0,1,2, \ldots$. Accordingly, the area of the first peak of the residence time distribution,

$$
\operatorname{RES}:=\int_{(1 / 2-\alpha) \tau}^{(1 / 2+\alpha) \tau} \mathrm{d} t_{\text {res }} P\left(t_{\text {res }}\right),
$$

has been proposed as a sensible quantifier of bona fide SR $[2-4,9,10]$. In doing so, the normalization

$$
\int_{0}^{\infty} \mathrm{d} t_{\mathrm{res}} P\left(t_{\mathrm{res}}\right)=1
$$




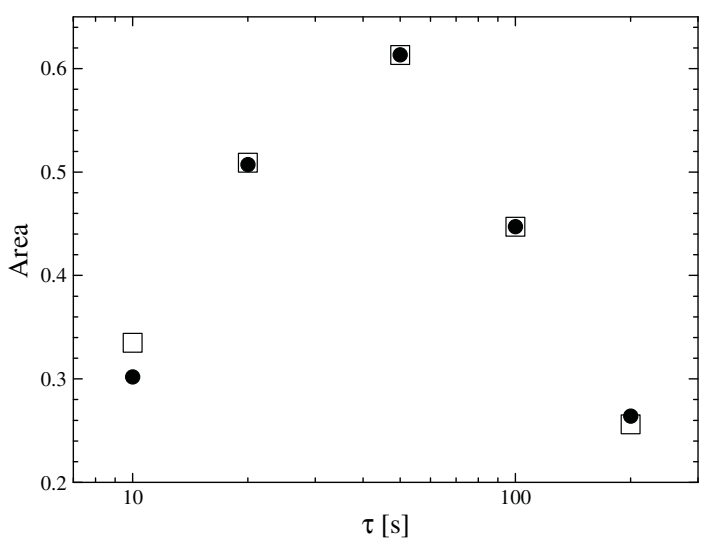

Figure 2. The same numerical data as in figure 1. Filled circles: area under the first peak of the residence time distribution RES from (47)-(49) versus driving period $\tau=2 \pi / \Omega$. Open squares: hysteresis loop area HLA from (25) versus driving period $\tau=2 \pi / \Omega$, scaled so that RES $(50 \mathrm{~s})=\operatorname{HLA}(50 \mathrm{~s})$.

is taken for granted and $\alpha$ may be any number in the interval $(0,1 / 2)$. Our standard choice in this paper is

$$
\alpha=0.2 \text {. }
$$

Indeed, RES in figure 2 exhibits the predicted resonance-type peak as a function of the driving period $\tau$.

Also shown in figure 1 are the hysteresis loops of the average coordinate $\langle x(t)\rangle$, parametrically plotted versus the external driving $f(t)$, for the same driving periods $\tau$ as the residence times from figure 1. The corresponding hysteresis loop areas (25) are depicted in figure 2. The quite remarkable agreement (up to a constant scaling factor) between these hysteresis loop areas and the area of the first peak of the residence time distribution (47) is further discussed in section 6.

From the very good quantitative agreement of these theoretical predictions with the experimental findings that will be demonstrated in the subsequent section 5 , we conclude that our model (35), (38)-(45) indeed describes the experimental reality reasonably well.

\section{Experimental system and results}

\subsection{Experimental set-up}

The situation discussed above was realized experimentally with colloidal particles suspended in water, a system for which the dynamics are well described by Brownian motion. Doublewell potentials were created and modulated using the principle of optical tweezers [27]. In our experiment we used two acousto-optic deflectors and a laser beam time-shared at a switching rate of $50 \mathrm{kHz}$ to create multiple optical traps with well defined positions and intensities. Because this timescale is significantly faster than any relaxation times in our system the colloidal particles felt quasi-static potentials. Due to the vertical light pressure acting on the particles, their motion was confined to a plane close to the lower surface of our sample cell. A colloidal particle fluctuating in a double-well potential was imaged onto a CCD camera. Images were recorded at a rate of $20 \mathrm{frames} \mathrm{s}^{-1}$ and later analysed to extract particle trajectories. Figure 3 illustrates the experimental set-up described in more detail in [28]. 

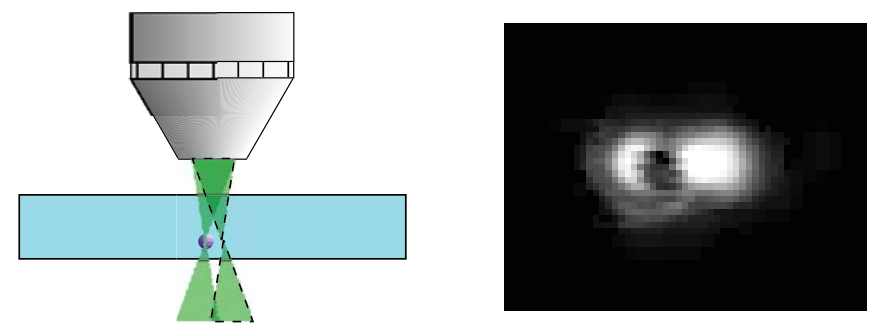

Figure 3. Left: schematic picture of our experiment. A laser beam is focused using a microscope objective to act as optical tweezers. The dashed line indicates the position of the laser beam about $20 \mu$ s later. Right: CCD camera image of a particle in a double-well potential.

(a)

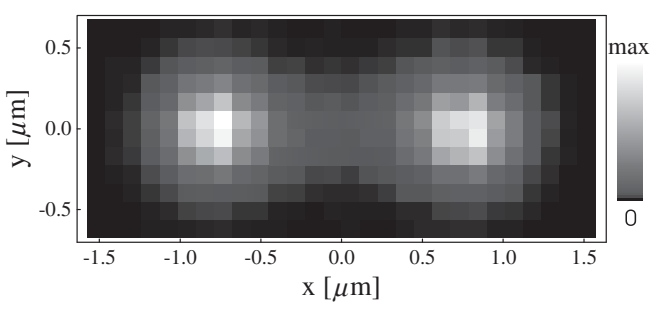

(b)

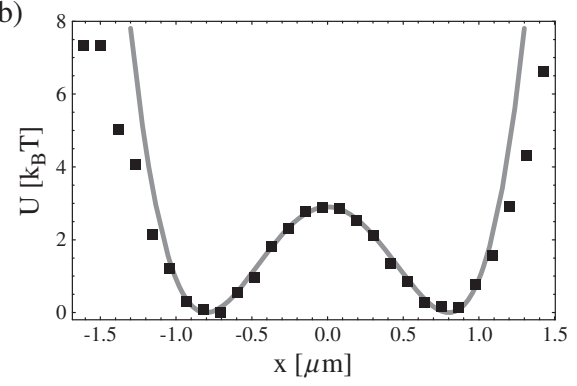

Figure 4. (a) Two-dimensional probability distribution of a colloidal particle fluctuating in a double-well potential. (b) Cross section at $y=0 \mu \mathrm{m}$ of the potential obtained from the probability distribution shown in (a) (symbols) and the potential $U(x)=2.9 k_{\mathrm{B}} T\left[(x / 0.8 \mu \mathrm{m})^{2}-1\right]^{2}(\mathrm{cf}(38)$ (40)) (line).

\subsection{Determination of model parameters}

Our experimental results were obtained using silica particles of diameter $2 r=(1.57 \pm$ 0.06) $\mu \mathrm{m}$. Our double-well potential had an inter-well distance of $2 x_{0}=(1.6 \pm 0.1) \mu \mathrm{m}$ (cf (39)) and a barrier height in the unmodulated case of $\Delta U=(2.9 \pm 0.3) k_{\mathrm{B}} T(\mathrm{cf}(40))$. These parameters were determined from the Boltzmann distribution sampled by a particle in the static double-well potential under the influence of thermal noise at room temperature (41) as shown in figure 4(a). The symbols in figure 4(b) show a cross section of the resulting potential $U(x, y)$ along the $y$-axis (connecting the extrema of the potential). For comparison we plotted the function $U(x)=2.9 k_{\mathrm{B}} T\left[(x / 0.8 \mu \mathrm{m})^{2}-1\right]^{2}$ (cf (38)-(40)). In the central part, the potential is very well described by this function, while deviations occur in the outer regions. The latter is due to the fact that far from the centre the light distribution, which can be approximated by the sum of two Gaussians, deviates significantly from the quartic form assumed in (38). However, it should be emphasized that the particle hopping dynamics, i.e. the Kramers time, are essentially determined by the potential shape in the vicinity of its extrema, where the agreement with (38) is indeed very good.

The double-well potential was modulated sinusoidally by varying the light intensities in the two traps in counterphase. The sine was sampled in 40 steps of length $\tau / 40$ (where $\tau=2 \pi / \Omega$ denotes the period of modulation) with a maximum change in intensity of $\pm 16 \%$. From this intensity modulation we estimated the modulation amplitude of the double-well potential in (35) to be $A=(1.9 \pm 0.5) k_{\mathrm{B}} T / x_{0}(\mathrm{cf}(42))$. 


\subsection{Results and discussion}

To compare our data with the simulations presented in section 4 we determined the residence time distributions and hysteresis loops from the $x$-components of the measured trajectories. This reduction to one dimension is justified a posteriori by the good agreement between the results of the one-dimensional simulation and our experimental measurements. For the residence time distributions we introduced the same thresholds (46) as in the simulations and adopted the same definition for the area of the first peak (47)-(49).

Figure 5 shows the residence time distributions and hysteresis loops for five driving periods $\tau=10,20,50,100$ and $200 \mathrm{~s}$. From an exponential fit to the residence time distribution for a particle fluctuating in the unmodulated double-well potential we find the Kramers time for our system to be $(22 \pm 4) \mathrm{s}$, which agrees well with the structure of the residence time distributions in figure 5. For $\tau=50 \mathrm{~s}$, corresponding roughly to twice the Kramers time, the residence time distribution exhibits primarily one peak centred at $t_{\text {res }}=25 \mathrm{~s}$. For shorter modulation periods $\tau$ we find multiple peaks at odd multiples of half the modulation period because the particle cannot follow the potential modulation. For longer modulation periods we observe an increasing number of jumps at short times because the probability for jumps from the deeper to the shallower well increases with increasing modulation period. Comparing the experimental residence time distributions in figure 5 to the simulated ones in figure 1 we find good qualitative agreement.

The right-hand column in figure 5 shows the hysteresis loops corresponding to the residence time distributions on the left-hand side. The average position $\langle x(t)\rangle$ entering the hysteresis loops has been evaluated from the $x$-component of the experimentally observed trajectories according to

$$
\langle x(t)\rangle:={ }_{N}^{1} \sum_{n=1}^{N} x(t+n \tau) .
$$

Due to ergodicity reasons, the discrete-time average in (50) is indeed equivalent to the ensemble-average $\langle x(t)\rangle$ used in the theoretical sections in the limit $N \rightarrow \infty$. In the experiment the measurement time was $85 \mathrm{~min}$ for each $\tau$ leading to approximately $N=500$ periods for $\tau=10 \mathrm{~s}$ and about $N=25$ for $\tau=200 \mathrm{~s}$; the small number of periods available for $\tau=200 \mathrm{~s}$ explains the steplike structure in this hysteresis loop.

These experimental hysteresis loops are in close agreement with the simulated ones shown in figure 1. To understand the shape of the hysteresis loops for the different modulation periods $\tau$ we look first at three extreme situations.

(1) The modulation period is extremely short. In this case the particle feels a symmetric average potential and $\langle x(t)\rangle$ will be zero for all values of $\cos (\Omega t)$.

(2) The modulation period is approximately twice the system Kramers time. Here, the particle follows the modulation and generally jumps twice per period. Typically the particle jumps at the quarter modulation period when the potential changes from the symmetric state to the maximally tilted one and remains in the deeper well until the potential is tilted towards the other side. This results in a hysteresis loop of maximum area tilted with a positive slope.

(3) The modulation period is much longer than the Kramers time (adiabatic limit). For an infinitely long modulation period the particle samples the potential for each step in the modulation and there is a unique $\langle x(t)\rangle$ corresponding to each value of $\cos (\Omega t)$. In this case the hysteresis loop degenerates into one single curve reaching from $-x_{0}$ to $x_{0}$. This dependence of the hysteresis loops on the modulation period can be observed in our experimental data. For $\tau=10 \mathrm{~s}\langle x(t)\rangle$ remains about zero throughout the range of 

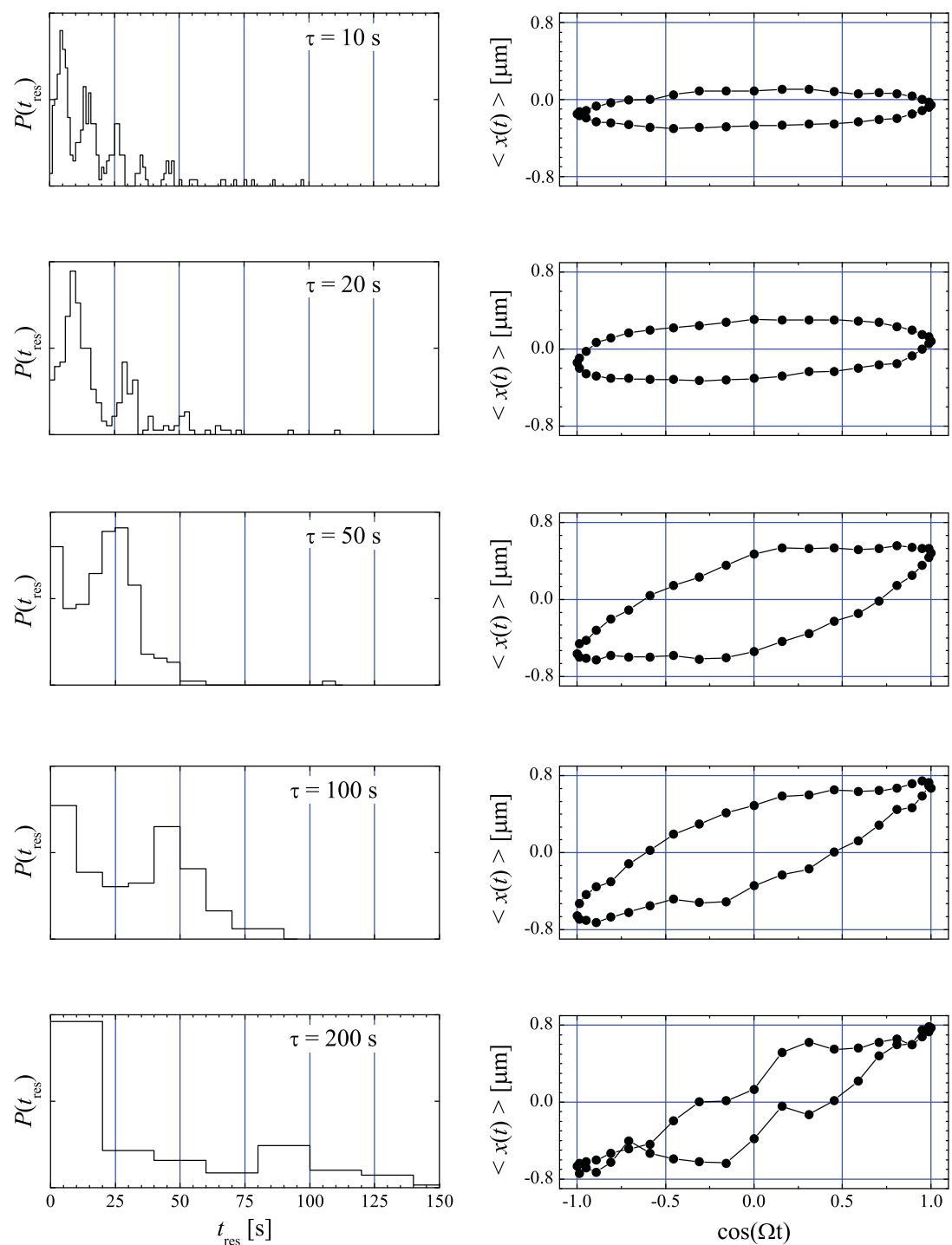

Figure 5. Experimental residence time distributions $P\left(t_{\text {res }}\right)$ (histograms) and hysteresis loops (parametric plots of $\langle x(t)\rangle$ versus $\cos (\Omega t)$ with $t$ as parameter) for the same driving periods $\tau$ as in figure 1. The measurement time was $85 \mathrm{~min}$ for each of the driving periods.

$\cos (\Omega t)$. The small shift of the hysteresis loop for $\tau=10 \mathrm{~s}$ is due to the particle spending more time in the left potential well than the right one in this particular measurement. For $\tau=50 \mathrm{~s}$ the hysteresis loop has maximum area and is clearly tilted with a positive slope; however, compared to the simulations, the experimental loop is slightly flattened. Finally, for $\tau=200 \mathrm{~s}$ the hysteresis loop shows an apparent tendency towards one single curve.

The dependence of the hysteresis loop areas and the first peaks in the residence time distributions on modulation period $\tau$ can be estimated from figure 5. These quantities are plotted in figure 6. Filled circles correspond to the area under the first peak of the residence time distribution RES while open squares are the hysteresis loop area HLA determined by 


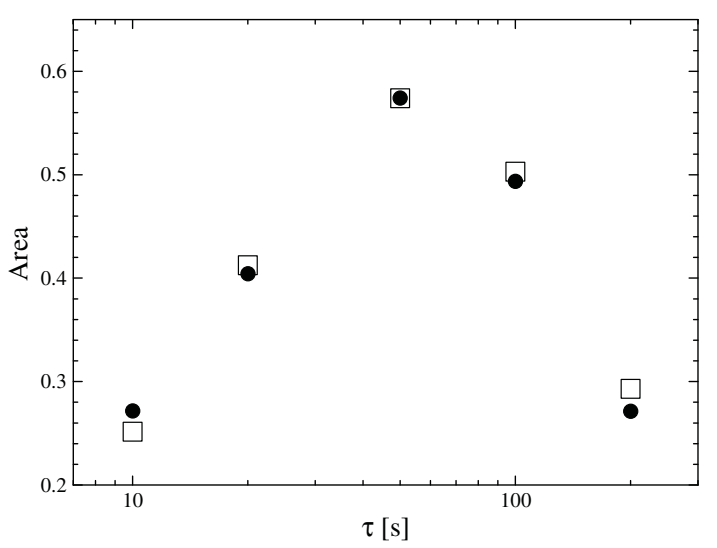

Figure 6. The experimental data as in figure 5. Filled circles: area under the first peak of the residence time distribution RES from (47)-(49) versus driving period $\tau=2 \pi / \Omega$. Open squares: hysteresis loop area HLA from (25) versus driving period $\tau=2 \pi / \Omega$, scaled so that RES $(50 \mathrm{~s})=\operatorname{HLA}(50 \mathrm{~s})$.

interpolating the measured values linearly before integration. The HLA was scaled in such a way that the peak values of the area under the first peak and the hysteresis loop area coincide, $\operatorname{RES}(\tau=50 \mathrm{~s})=\operatorname{HLA}(\tau=50 \mathrm{~s})$. It is apparent that both criteria agree very well for our measurements as is the case for the simulations (cf figure 2). Both curves show a clear peak at $\tau=50 \mathrm{~s}$, which we expect based on both the system Kramers time and the residence time distributions and hysteresis loops in figure 5. Comparing the experimental curves with the simulations in figure 2 the only apparent difference is in the areas for $\tau=20$ and $100 \mathrm{~s}$, about which one can only speculate at this point. Most likely the limited statistics of the experiment plays a role while also the parameters used for the simulation may differ slightly from the true values.

\section{Summary and conclusions}

The main theme of our present work is the comparison of different quantifiers for SR.

A first important result is represented by the relation (36) between three common such quantifiers, namely signal-to-noise ratio, spectral power amplification, and hysteresis loop area (HLA), which is valid for an extremely general class of systems under the influence of a weak, harmonic driving. In fact, this is essentially the only mathematical relation between different SR quantifiers known to us, in spite of the fact that the underlying qualitative picture is the same for all of them.

In particular, for the most common quantifier in the context of bona fide SR, namely the area under the first peak of the residence time distribution (RES), no quantitative relation with any other SR quantifier has been reported in the literature we know, nor were we able to derive such a relation even for very simple special models.

Therefore, the second main result of our present paper, namely the surprisingly good agreement of the bona fide SR quantifiers HLA and RES in figures 2 and 6, represents a challenging open problem for future theoretical studies.

The third central point of our present work is the very good quantitative agreement between the simple theoretical model in section 4 and the experimental system from section 5 . 


\section{Acknowledgments}

We thank Peter Talkner and Peter Jung for helpful remarks. This work was supported by the Deutsche Forschungsgemeinschaft under RE 1344/3-1 and BE 1788/4-1, and by the Alexander von Humboldt-Stiftung.

\section{References}

[1] Gammaitoni L, Hänggi P, Jung P and Marchesoni F 1998 Rev. Mod. Phys. 70223

[2] Choi M H, Fox R F and Jung P 1998 Phys. Rev. E 576335

[3] Giacomelli G, Marin F and Rabbiosi I 1999 Phys. Rev. Lett. 82675

[4] Marchesoni F, Gammaitoni L, Apostolico F and Santucci S 2000 Phys. Rev. E 62146

[5] Evstigneev M, Pankov V and Prince R H 2001 J. Phys. A: Math. Gen. 342595

[6] Evstigneev M, Pankov V and Prince R H 2002 Phys. Rev. Lett. 88240201

[7] Talkner P 2003 Physica A 325124

[8] Evstigneev M, Reimann P, Pankov V and Prince R H 2004 Europhys. Lett. 657

[9] Gammaitoni L, Marchesoni F and Santucci S 1995 Phys. Rev. Lett. 741052

[10] Berglund N and Genz B 2005 Europhys. Lett. 701

[11] Mahato M C and Shenoy S R 1994 Phys. Rev. E 502503

[12] Phillips J C and Schulten K 1995 Phys. Rev. E 522473

[13] Thorwart M and Jung P 1997 Phys. Rev. Lett. 782503

[14] Mahato M C and Shenoy S R 1997 Phys. Rev. E 556266

[15] Sides S W, Rikvold P A and Novotny M A 1998 Phys. Rev. Lett. 81834 Sides S W, Rikvold P A and Novotny M A 1998 Phys. Rev. E 576512

[16] Thorwart M, Reimann P, Jung P and Fox R F 1998 Chem. Phys. 23561

[17] Iwai T 2001 Physica A 300350

[18] Lim J S, Choi M Y and Kim B J 2003 Phys. Rev. B 68012501

[19] Dan D and Jayannavar A M 2005 Physica A 345404

[20] Kubo R 1966 Rep. Prog. Phys. 29255

[21] Chandler D 1987 Introduction to Modern Statistical Mechanics (New York: Oxford University Press)

[22] Landau L and Lifshitz E 1980 Statistical Physics (Oxford: Pergamon)

[23] Jung P and Hänggi P 1989 Phys. Rev. A 448032

[24] Casado-Pascual J, Denk C, Gomez-Ordonez J, Morillo M and Hänggi P 2003 Phys. Rev. E 67036109

[25] Casado-Pascual J, Gomez-Ordonez J, Morillo M and Hänggi P 2003 Phys. Rev. E 68061104

[26] Hänggi P, Talkner P and Borkovec M 1990 Rev. Mod. Phys. 62251

[27] Ashkin A 1970 Phys. Rev. Lett. 24156

[28] Babič D, Schmitt C, Poberaj I and Bechinger C 2004 Europhys. Lett. 67158 